\title{
DESENVOLVIMENTO E IMPLEMENTAÇÃO DE SISTEMA ELETRÔNICO DE ALERTA DE CHUVAS PARA ÁREAS DE RISCO
}

\author{
Eliel da Silva Macena \\ Graduando em Engenharia Elétrica pelo Centro Universitário Augusto Motta (UNISUAM) \\ Rio de Janeiro, RJ, Brasil \\ eliel.macena@gmail.com \\ Antônio José Dias da Silva \\ Mestre em Sistemas de Informação pela Universidade Federal do Rio de Janeiro (UFRJ) \\ Professor da Faculdade Cesgranrio - FACESG \\ Rio de Janeiro, RJ, Brasil \\ antoniojose@ cesgranrio.org.br \\ Geraldo Motta Azevedo Junior \\ Doutor em Engenharia Elétrica pela Universidade Federal do Rio de Janeiro (COPPE/UFRJ) \\ Professor do Centro Universitário Augusto Motta - UNISUAM \\ Rio de Janeiro, RJ, Brasil \\ geraldomotta@unisuam.edu.br \\ André Luis da Silva Pinheiro \\ Doutor em Engenharia Nuclear pela Universidade Federal do Rio de Janeiro (UFRJ) \\ Professor do Centro Universitário Augusto Motta - UNISUAM \\ Rio de Janeiro, RJ, Brasil \\ apinheiro99@gmail.com \\ Vinícius Coutinho de Oliveira \\ Mestre em Engenharia Elétrica pelo Yokohama National University (YNU) - Japão. \\ Professor do Centro Universitário Augusto Motta - UNISUAM \\ Rio de Janeiro, RJ, Brasil \\ vini.coutinho@gmail.com
}

\section{RESUMO}

Evitar acidentes, garantir o bem-estar e salvar vidas, tem sido uns dos principais desafios da engenharia moderna. $\mathrm{O}$ objetivo deste trabalho foi a criação e implementação de um modelo de pluviômetro, de baixo custo, utilizando um modelo digital, artesanal com comparadores lógicos programáveis e um circuito eletrônico de autoria própria, que associado a outros instrumentos de sinalização e alarme, venha colaborar na prevenção de acidentes oriundos de altos índices pluviométricos, em comunidades de baixa renda. Após montado e testado verificou-se que o protótipo atendeu satisfatoriamente a que se propôs.

Palavras-chave: Sensor, Automatizado, Pluviômetro, Eletrônica, Amplificador.

\begin{abstract}
Avoiding accidents, guaranteeing well-being and saving lives, has been one of the main challenges of modern engineering. The objective of this work was the creation and implementation of a low cost rain gauge model, using a digital model, handcrafted with programmable logic comparators
\end{abstract}


and an electronic circuit of its own authorship, which associated with other signaling and alarm instruments, come and collaborate in prevention of accidents resulting from high rainfall, in lowincome communities. After assembled and tested it was found that the prototype satisfactorily met what was proposed.

KeyWords: Sensor, Automated, Rain Gauge, Electronics, Amplifier.

\section{INTRODUÇÃO}

Sabe-se que a chuva é fundamental para a vida humana no planeta Terra, sendo responsável por incentivar o crescimento de plantações, pela dinâmica de rios e mares, e por ser essencial para a alimentação de animais selvagens e domésticos, além de ser a principal abastecedora dos lençóis freáticos e pelo abastecimento de biomas. (MARCUZZO E CARDOSO, 2012).

Porém, a chuva também pode ser fundamental para a destruição de plantações, cheias de rios e desastres urbanos, sendo esse último cada vez maior frente ao crescimento desenfreado da população, no que tange aos desabamentos de construções irregulares, geralmente em áreas de encostas provocadas por erosões, alagamento, entre outros, quando em excesso, destacando-se as fortes chuvas tropicais, frequentes nas cidades brasileiras e deflagradoras de calamidades públicas, causando alto impacto econômico e psicossocial para toda sociedade (OSCAR, 2018).

Contudo, se tais desastres naturais forem previstos com antecedência suficiente, esses danos podem ser minimizados, a partir de informações meteorológicas, através de modelos matemáticos da atmosfera e equipamentos, contribuindo assim para sistemas de alerta (ENGELBRECHT et al., 2010).

Logo, o objetivo desse estudo foi buscar uma forma de minimizar o problema das áreas de risco que sofrem com as tragédias causadas por grandes chuvas, propondo a implementação de um sistema automático de monitoramento pluviométrico (BATISTEL, CAUVILLA E LODI, 2017), utilizando um pluviômetro do modelo digital, artesanal que pode ser comparado com o modelo Ville de Paris (modelo mais simples para confecção de um pluviômetro artesanal). Projetado com o intuito de ser um sistema de baixo custo e acessível às comunidades de baixa renda, pois segundo (ARAUJO et al, 2014) um sistema desse tipo pode ser implementado com um investimento de até $65 \%$ mais barato que uma estação meteorológica profissional.

Dessa forma, este protótipo foi constituído com comparadores lógicos programáveis e um circuito eletrônico de autoria própria com a finalidade de corroborar com o sistema de sinalização, através do envio de informação e acionamentos de sirenes espalhadas estrategicamente nos locais de risco, bem como tornar esse recurso acessível a toda comunidade. 


\section{DESENVOLVIMENTO}

\subsection{Funcionamento do Pluviômetro}

O pluviômetro é um instrumento meteorológico que é comumente utilizado para se obter medidas de precipitações, isto é, elementos líquidos ou sólidos (chuvas, granizo ou neve) que caem do céu. Diferente do pluviógrafo, que é um instrumento com as mesmas características do pluviômetro, mas com o intuito de registrar o tempo. O pluviômetro dispõe de uma escala para indicar, a cada milímetro, o volume em litros de água que foram concentrados em um metro quadrado por um determinado tempo. "Quando se diz que houve 10 milímetros de chuva, se quer dizer que esta é a altura média de água a partir do chão em uma localidade, em um período de tempo (1 milímetro de chuva equivale a 1 litro de água por metro quadrado) " (ARAUJO E GONÇALVES, 2014).

\subsection{Tipos de Pluviômetros}

Existem diversas formas e tipos de ferramentas para se obter medidas de precipitações pluviométricas e dados meteorológicos em campo. O modelo de pluviômetro mais utilizado em estações meteorológicas é o pluviômetro de báscula. O pluviômetro de báscula se constitui de um funil que recebe a água da chuva, e transporta a água para um mecanismo basculante semelhante a um monjolo duplo (engenho rudimentar utilizado para acionar moinhos com o movimento da água) que, quando cheio um dos lados, tende a desequilibrar, tombando para o lado que estava com a água da chuva, despejando a água e começando um novo ciclo para o outro lado. Cada movimento feito pela báscula é contabilizado no sistema como uma soma de medidas iguais e relacionado durante um determinado espaço de tempo.

Este modelo de pluviômetro permite uma leitura das medidas mais precisas e confiáveis. Uma vez que a báscula somente se movimenta com um determinado peso exercido pelo volume de água, gerando um pulso eletrônico enviado ao sistema de data logger implementado ao conjunto de sensores, que registra o momento em que o reservatório tomba, calculando o volume de precipitação que cai durante um determinado tempo. Cada pulso gerado pelo sensor representa 1 mm de altura da chuva. O sistema de Báscula necessita de uma calibração e uma manutenção preventiva periódica para apresentar resultados mais exatos e confiáveis, devido a comuns defeitos que ocorrem durante a sua utilização e também devido a sua exposição ao tempo. De acordo com o relatório de calibração de Alexandre et al., 2006, muitos pluviômetros desse tipo padecem de erro. Além da inconstância de medidas feitas fisicamente, também existem erros tendenciosos que devem 
ser levados em consideração e calibrados antes da sua utilização. Erros sistêmicos que devem ser considerados como base padrão para tomada de decisão.

Outro tipo de pluviômetro muito comum e facilmente encontrado em estações meteorológicas é o Ville de Pari (que pode ser visto na figura 1). Este equipamento se constitui em um recipiente de forma cilíndrica e graduada, que permite a conferência das medidas manualmente e mantém a água da chuva em um reservatório para medições posteriores nos horários definidos pela Organização Mundial de Meteorologia (OMM), que estipula horários padrões para registro das medidas: 09:00, 15:00 e 21:00 (FERREIRA E FISCH, 2010). Este é o modelo de pluviômetro mais utilizado no Brasil. Ele tem a entrada receptora do funil padronizado de 400 centímetros quadrados e um reservatório com uma válvula de alívio para descarregar a água em uma proveta graduada para medição. Comumente, são fornecidas provetas especiais de 7, 10, 25 e 80 milímetros já graduados para este tamanho da área coletora, a fim de evitar os cálculos. Por ser de simples instalação e baixo custo, esse instrumento é ideal para implementação em locais de fins diversos e também é muito utilizado em instituições de ensino para fins de educação cientifica e geográfica, por ser simples de utilizar e complementar as aulas práticas de temas como consciência ambiental.

Figura 1 - Pluviômetro Ville de Paris

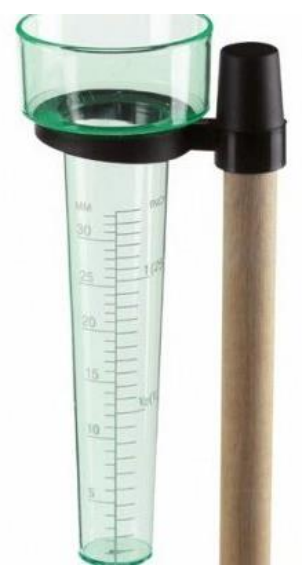

Fonte: Adaptado de http://lamiradaweb.com/w/sociales/

17-mm-la-lluvia-caida-hasta-el-momento

File:uploads/2016/09/pluviometro-458x400.jpg, acessado em 02/01/2019.

Até recentemente, a quantificação apresentada por esse instrumento dependia inteiramente de leitura manual, demandando uma mão de obra para leitura do pluviômetro. O Ville de Paris também pode apresentar erros de leitura por conta de evaporação da água com o decorrer do tempo, perdas nas paredes do recipiente e erros humanos como, leituras incorretas (erro de paralaxe), porém não se descarta a utilização de um sensor de nível para extração de dados de forma automatizada. 


\subsection{Simulação}

O protótipo do sistema de coleta pluviométrica deste trabalho, é constituído por um pluviômetro modelo Ville de Paris construído de forma artesanal com materiais de baixo custo e até mesmo reutilizados para baixar os custos de projeto da melhor formar possível, utilizando:

- $\quad$ Copo de medidas $500 \mathrm{ml}=(9 \mathrm{~cm}) \times 15 \mathrm{~cm}$ (reutilizado);

- $\quad$ Funil com diâmetro de entrada de $12,7 \mathrm{~cm}$ (reutilizado);

- $\quad$ Válvula solenoide $12 \mathrm{~V}(\mathrm{R} \$ 45,00)$;

- $\quad$ Sensor de nível de água FD10 (R \$9,90);

- $\quad$ Suporte de fixação alumínio (reutilizado);

- $\quad$ Circuito eletrônico (custo em torno de R $\$ 57,00$ ).

Para implementação do protótipo do presente projeto, é utilizado o sensor de nível de água FD10 (que pode ser visto na figura 2), que tem sua impedância variável de acordo com o nível da água no recipiente do pluviômetro. Um sensor de baixo custo, que pode ser facilmente encontrado em lojas de eletrônica em geral, e com alta eficiência. A placa do sensor é tratada quimicamente para melhor durabilidade, para aumentar sua condutividade elétrica e sua resistência à corrosão. Este sensor é alimentado por uma tensão contínua na faixa de $3 \mathrm{~V}$ a $5 \mathrm{~V}$ e com corrente de trabalho superior a $20 \mathrm{~mA}$ e com sua faixa de saída de sinal: $0 \mathrm{~V} \sim 3,2 \mathrm{~V}$ (de acordo com o nível de imersão do sensor na água).

Figura 2 - Sensor de Nível de Água FD10

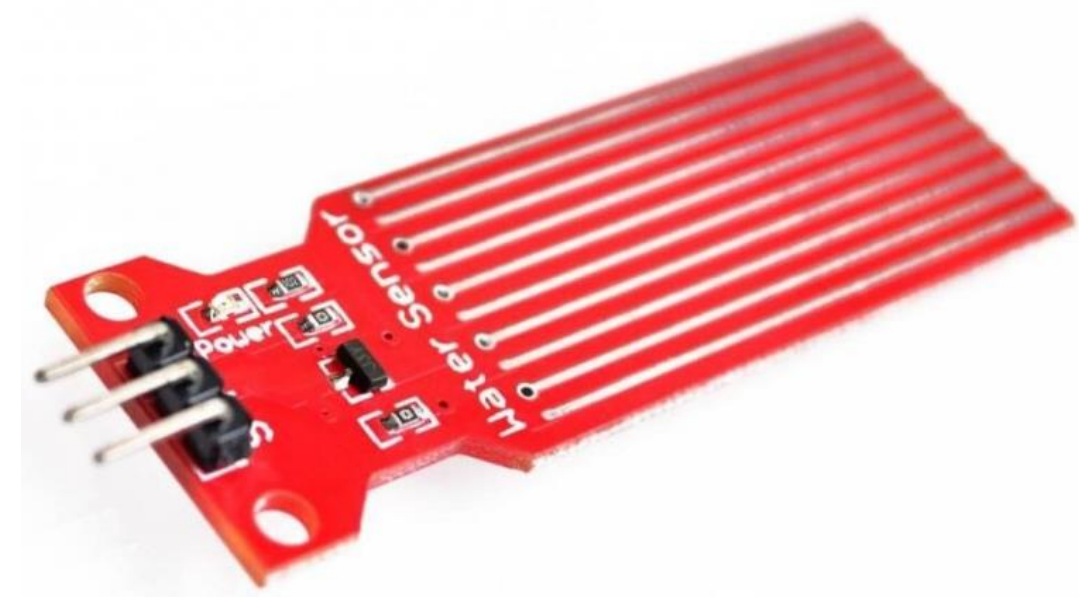

Fonte: Adaptado de https://www.daeletrica.com.br/sensor-de-nivel-e-profundidade-da-agua File: sensor-nivel-de-agua-800x800.jpg, acessado em 02/01/2019. 
O sensor responsável pelo monitoramento do nível pluviométrico está conectado ao circuito eletrônico específico e autoral, composto basicamente por amplificadores operacionais do modelo LM111 que é projetado na configuração de um circuito comparador de janela e por divisores de tensão que definem os quatro níveis de criticidade apresentados, para então enviar o pulso elétrico necessário para ativação do alarme. O sistema de alarme é configurado em 6 etapas distintas, para cada etapa, uma faixa de tensão diferente. Conforme mostrado na tabela 1.

Tabela 1: Tabulação e comandos do sensor

\begin{tabular}{|c|c|c|c|}
\hline Time & $\begin{array}{l}\text { Corrente no sensor } \\
\qquad\left(\mathrm{I}_{\mathrm{S}}, \mathrm{em} \mathrm{mA}\right)\end{array}$ & $\begin{array}{c}\text { Tensão aplicada nos } \\
\text { comparadores }\left(\mathrm{V}_{\mathrm{S}}, \text { em } \mathrm{V}\right)\end{array}$ & Ação do circuito \\
\hline $0 \leq X<5$ & $0 \leq$ Is $<0,5$ & $0 \leq \mathrm{V}_{\mathbf{S}}<0,287$ & $\begin{array}{c}\text { Inibe o temporizador de } \\
\text { solenoide. }\end{array}$ \\
\hline $5 \leq X<15$ & $0,5 \leq \mathrm{Is}<2,5$ & $0,28 \leq \mathrm{Vs}_{\mathrm{s}}<0,862$ & $\begin{array}{c}\text { Aciona o temporizador } \\
\text { de solenoide. }\end{array}$ \\
\hline $15 \leq X<20$ & $2,5 \leq \mathrm{Is}<4$ & $0,862 \leq \mathrm{VS}_{\mathbf{S}}<1,15$ & $\begin{array}{l}\text { Aciona o sinal da sirene } \\
\text { de modo intermitente. }\end{array}$ \\
\hline $20 \leq X<35$ & $4 \leq \mathrm{Is}<20$ & $1,15 \leq \mathrm{Vs}_{\mathrm{s}}<2,02$ & $\begin{array}{l}\text { Aciona o sinal da sirene } \\
\text { de modo contínuo. }\end{array}$ \\
\hline 35 & 20 & 2,02 & $\begin{array}{l}\text { Gera um sinal de by- } \\
\text { pass no temporizador } \\
\text { de solenoide. }\end{array}$ \\
\hline
\end{tabular}

Fonte: Autores

Através do método de interpolação, é possível estipular os valores de corrente aproximados para os outros valores de nível da água no pluviômetro (5 mm e $35 \mathrm{~mm}$ ). Para cada um dos 4 comparadores, foi calculado um divisor de tensão com fins de se formar sinais de referência de acordo com os valores de corrente encontrados. Assim, a partir desses dados, foi inserido o valor das tensões aplicadas nos comparadores e suas respectivas reações perante o circuito, conforme é ilustrado na Figura 3, Cada LED nas saídas VF, indicam qual circuito está sendo acionado no momento de acordo com o sinal que o circuito está recebendo. 
Figura 3 - Circuito Comparador de Janela do Sensoriamento

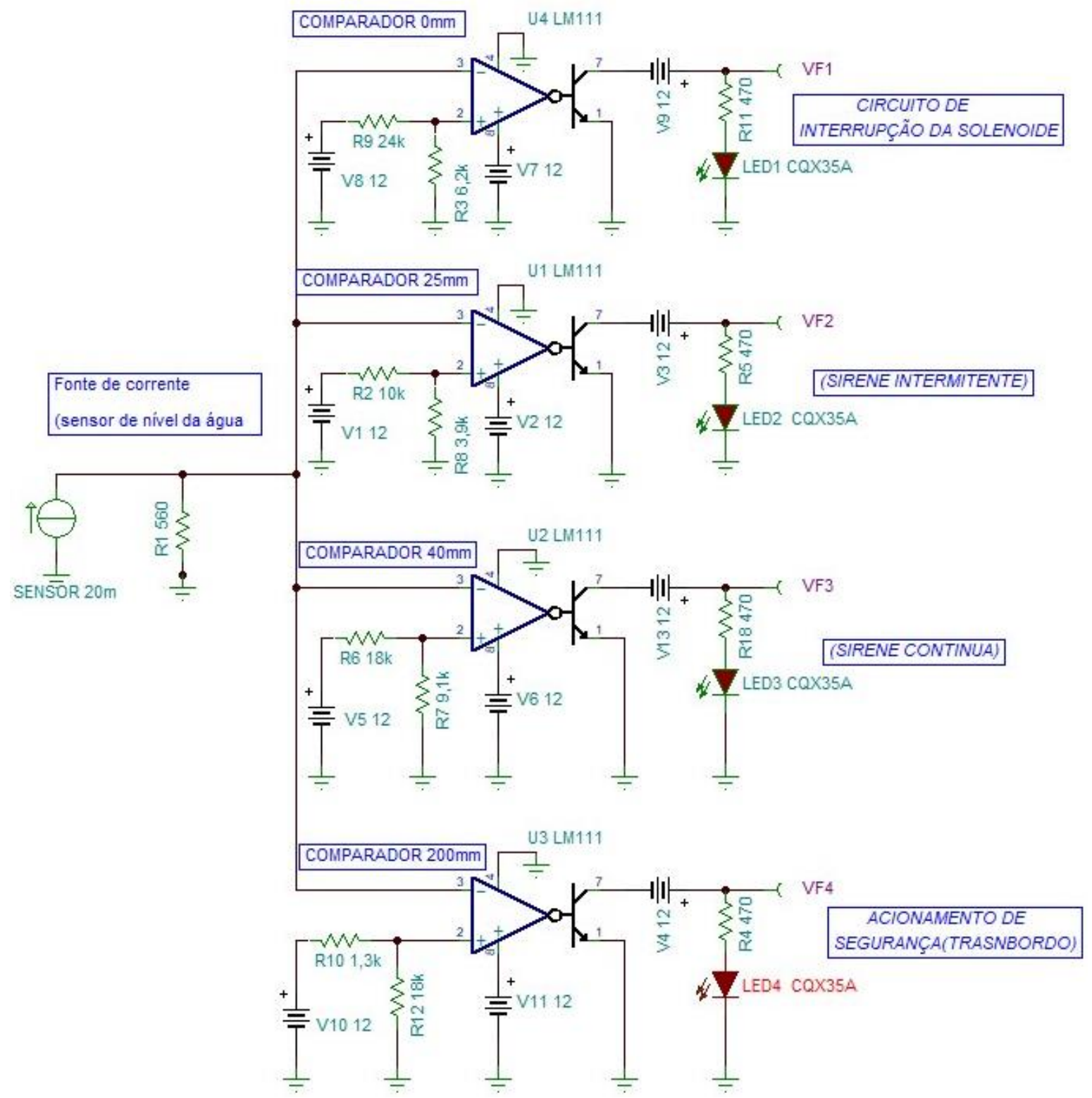

Fonte: Autores

O projeto do pluviômetro conta inicialmente, com a utilização do amplificador comparador LM111, que tem um tempo rápido de resposta (cerca de 165ns), pode ser alimentado por uma faixa de tensão que corresponde entre $-15 \mathrm{~V}$ e $+15 \mathrm{~V}$, com saída transistorizada compatível com a maioria dos circuitos CMOS e TTL, capaz de comandar lâmpadas e relés de até $50 \mathrm{~V}$ e $50 \mathrm{~mA}$. O comparador de tensão LM111 tem como encapsulamento, apenas um circuito por componente, e tem a disponibilidade de ajuste da corrente de offset máxima de 70 nA. Porém, esse modelo de comparador de tensão não é facilmente encontrado no mercado brasileiro.

Figura 4 - Circuito Simplificado do comparador LM111 


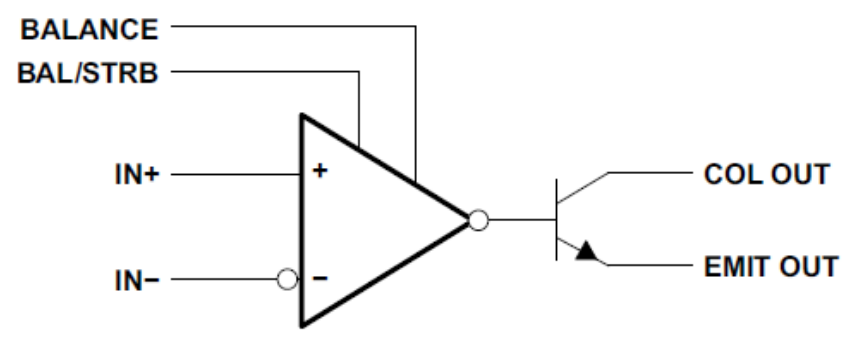

Fonte: Adaptado de http://www.alldatasheet.com/datasheet-pdf/ pdf/17868/PHILIPS/LM111.html, acessado em 02/01/2019.

O sensor previamente selecionado para a construção do protótipo é o sensor de nível de água analógico FD10, que durante uma bateria de testes, apresentou resultados que não estavam previstos anteriormente. A leitura dos níveis de água não é apresentada de forma linear e apresenta uma grande variabilidade de acordo com as impurezas na água da chuva ou da forma como a água é despejada no recipiente, portanto é necessário que seja feito uma compensação no circuito de referência dos comparadores.

Foram realizados testes com o sensor FD10 para calcular e definir, em volts, os pontos relativos aos acionamentos de criticidade e operação do pluviômetro de acordo com os valores obtidos na Tabela 1 anteriormente com seis variáveis relacionadas ao tipo de água aplicada e relacionada à forma como é inserida no recipiente do pluviômetro. Os testes tiveram os resultados obtidos no gráfico da Figura 5.

Figura 5 - Resultado dos Testes do Sensor de Nível Com Índices Lineares

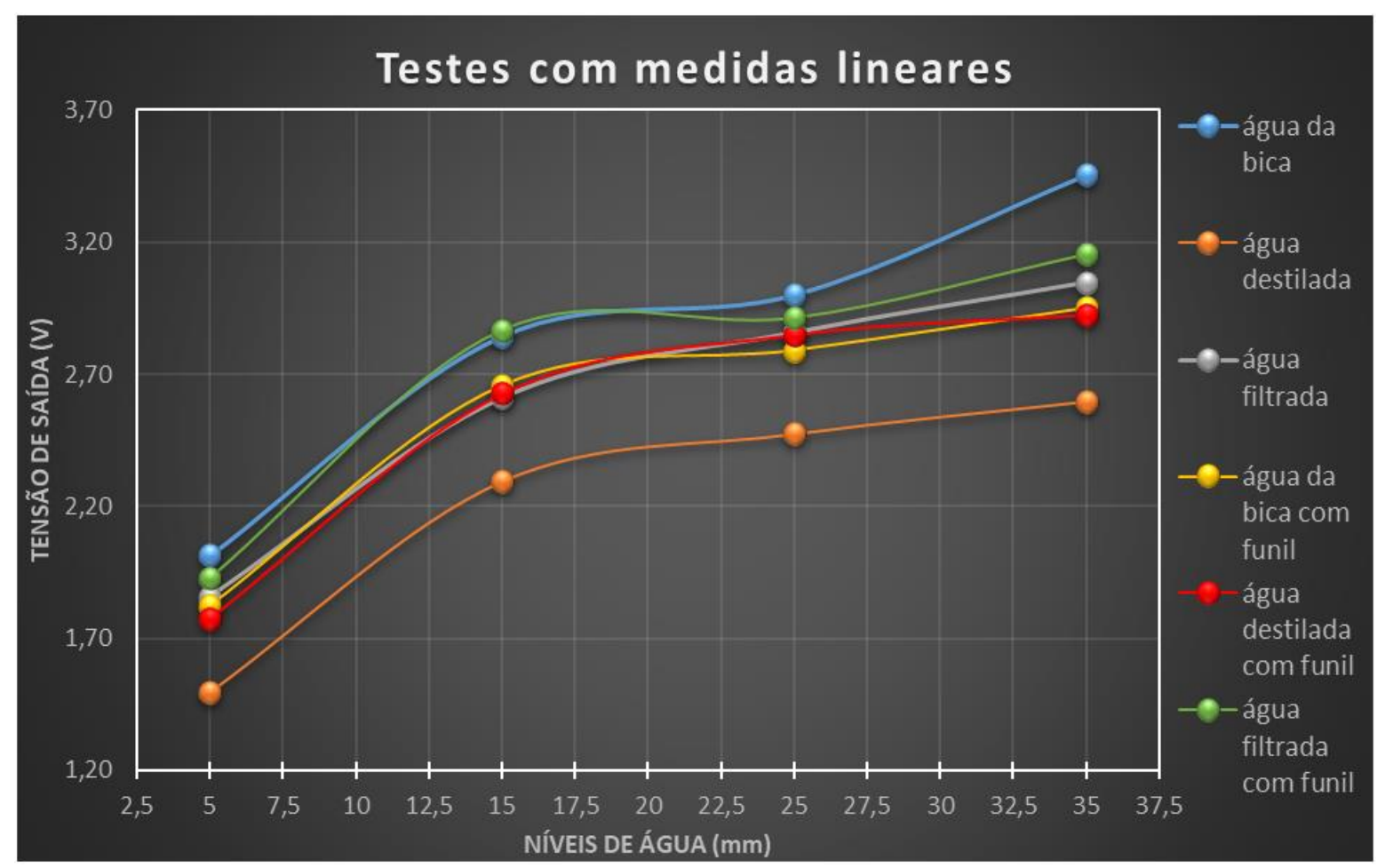


Com a escala de níveis do sensor definida entre 5 e $35 \mathrm{~mm}$ e com intervalos de $10 \mathrm{~mm}$ entre cada objetivo, os resultados não foram satisfatórios. Apesar de apresentar valores médios de tensão na saída, a partir das medidas de $25 \mathrm{~mm}$ os valores de tensão tendem a não se modificar, causando baixa confiabilidade na precisão do produto.

Foi realizado um novo teste do sensor, com os níveis de água sendo variados através de progressão geométrica, com a razão da progressão igual à 3, em níveis de diferenças. Foram obtidos os seguintes níveis pluviométricos: $3 \mathrm{~mm}, 9 \mathrm{~mm}, 21 \mathrm{~mm}$ e $39 \mathrm{~mm}$. Com esses níveis, com as mesmas condições e variáveis utilizadas anteriormente, foram obtidos os valores apresentados na Figura 6.

Figura 6 - Resultado dos Testes do Sensor de Nível Com Novos Índices

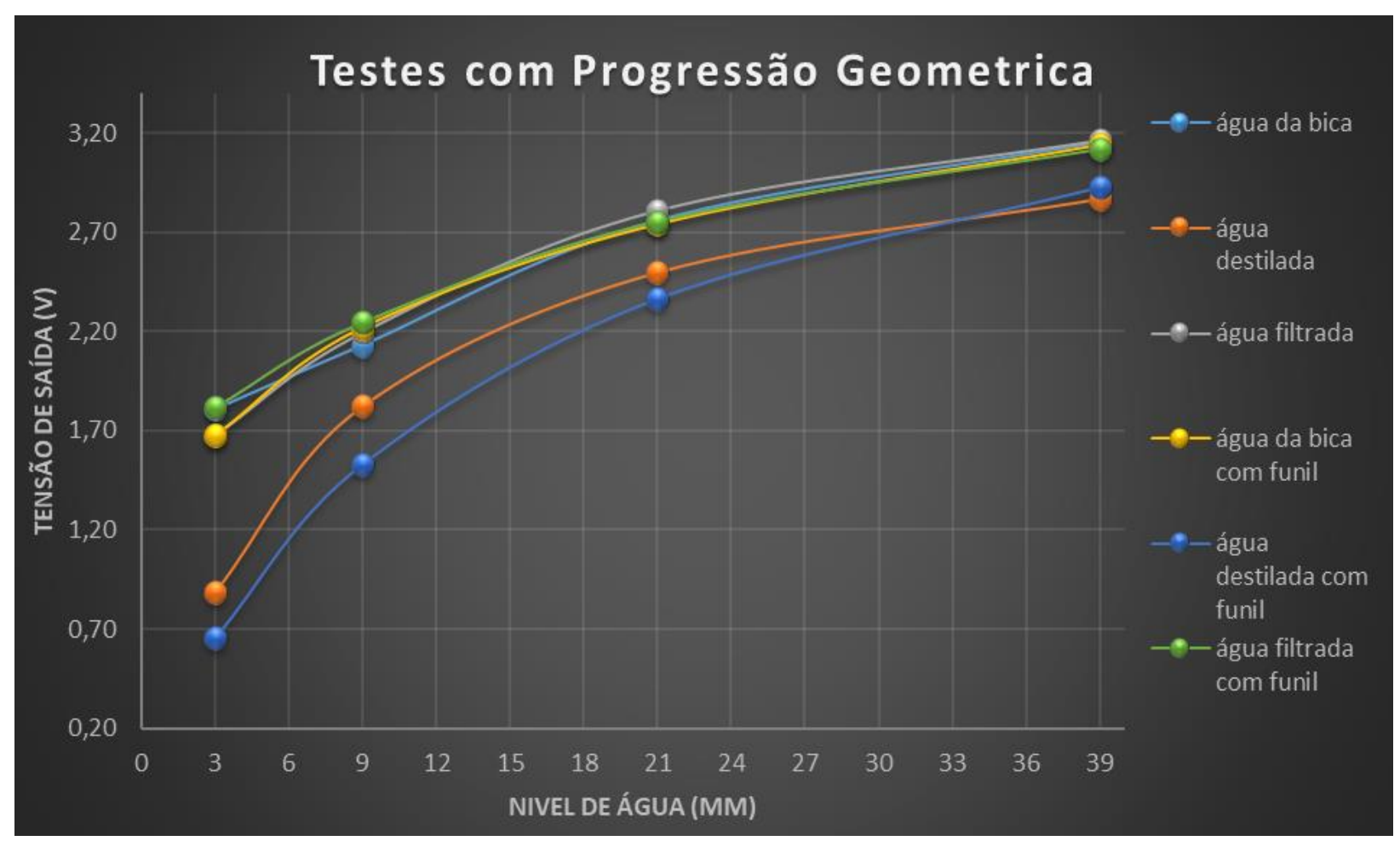

Fonte: Autores

Mesmo utilizando níveis pluviométricos adotados através de progressão geométrica ou linearmente, pode ser constatado que o sensor FD10 não é suficientemente adequado para a implementação de um projeto com precisão e sensibilidade de diferentes níveis de água. Conforme demonstrado nos gráficos da figura 5 e figura 6, a tensão média de saída apresentada pelo sensor mostra uma resolução menor conforme o nível de água no recipiente se eleva.

É perceptível que a sensibilidade do sensor é alterada em relação ao modo de inserção e tipo do líquido no recipiente. Uma vez que os níveis médios de tensão são mais precisos e mais lineares 
durante os testes realizados com água destilada despejada através de um funil. Enquanto a água comum (água da bica) despejada diretamente no recipiente, proporciona um comportamento totalmente diferente na tensão média do sensor.

Essa baixa precisão em níveis pluviométricos mais elevados, causados pela presença de impureza ou pela turbulência do despejar da água, pode ocasionar alarmes falsos, ou até mesmo o acionamento indevido da válvula solenoide do dreno com o nível pluviométrico ainda baixo, comprometendo assim, o funcionamento ideal do pluviômetro. Sendo considerada e necessária, a utilização de um sistema de leitura em paralelo para maior precisão de resultados e obtenção de uma margem de segurança.

Para implementação do circuito eletrônico impresso em placa, foi utilizado o sistema de criação e simulação de circuitos eletrônicos Protheus 8. Com auxílio de funções para confecções de trilhas e vasta biblioteca de componentes eletrônicos, foi possível realizar a simulação do circuito com níveis pluviométricos pré-definidos linearmente, definindo cada ação para sua respectiva situação. Foram utilizados alguns ajustes diante do projeto inicial para se adequar ao circuito físico, a fim de evitar ruídos no circuito e possibilitando ajustes finos nos sinais de referência dos comparadores de tensão. Foram acrescentados resistores ajustáveis do modelo Trimpot, em série com os divisores de tensão nos sinais de referência. Com essas adaptações, o circuito passa a ser considerado com a configuração da Figura 7.

Figura 7 - Plotagem do Circuito

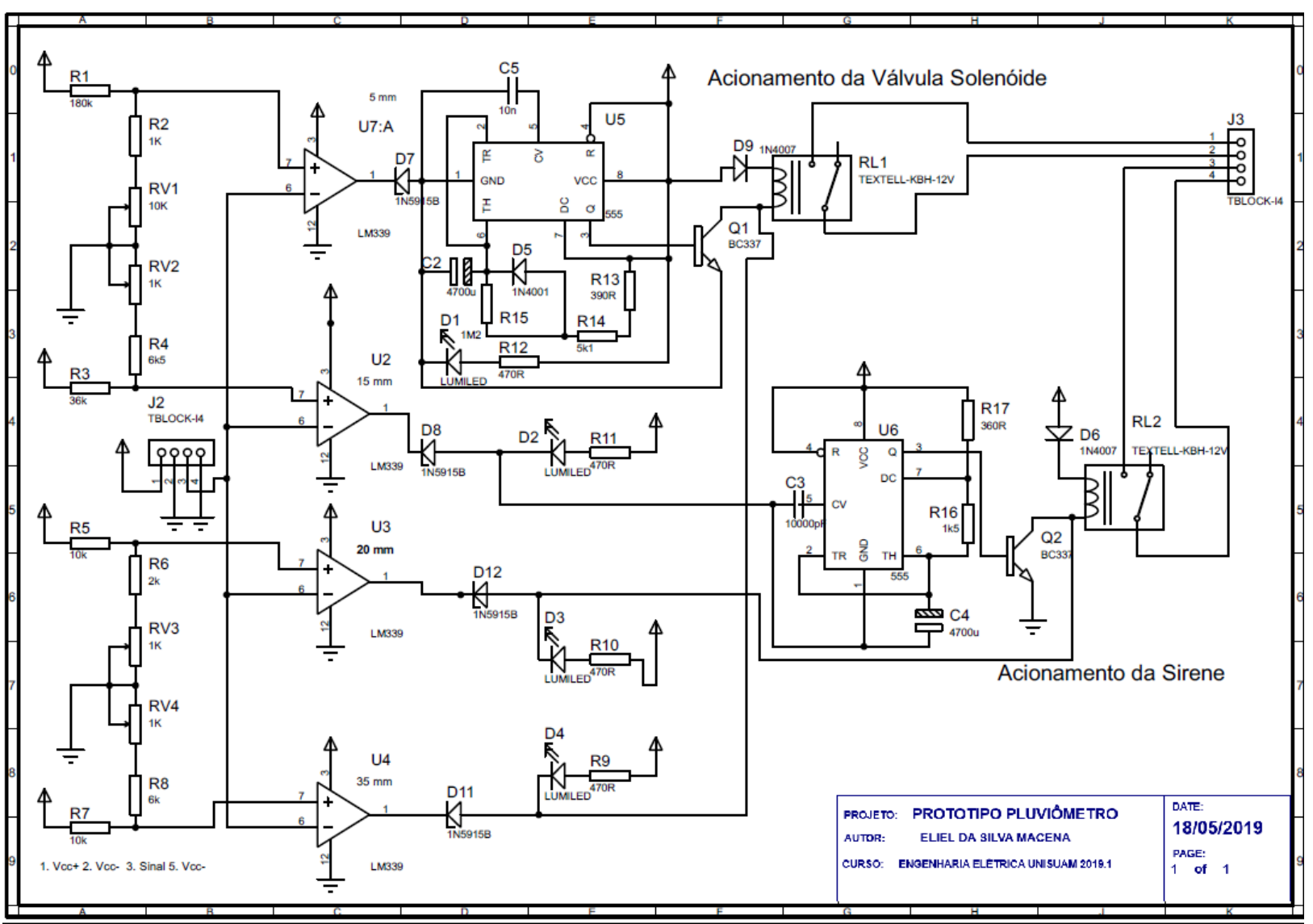


Foi feita a montagem em protoboard para testes do circuito em geral. Foram feitos testes do sensor de nível FD10 em conjunto com os circuitos de temporização do acionamento da válvula solenoide e do oscilador, responsável pelo modo intermitente da sirene, conforme mostrado na Figura 8. Como mencionado nos testes anteriores, o comportamento do sensor não foi satisfatório em relação à fidelidade de suas medições. Foram utilizados testes para ilustrar os devidos acionamentos visualmente.

Foram inseridos LEDs indicativos para uma melhor representação visual do sistema:

- $\quad$ LED amarelo: Nível de 3 mm;

- $\quad$ LED azul: Nível de $9 \mathrm{~mm}$;

- $\quad$ LED vermelho: Nível de $21 \mathrm{~mm}$;

- $\quad$ LED verde: Nível de $39 \mathrm{~mm}$;

- LEDs brancos: Sirene e válvula de escape.

Figura 8 - Pluviômetro

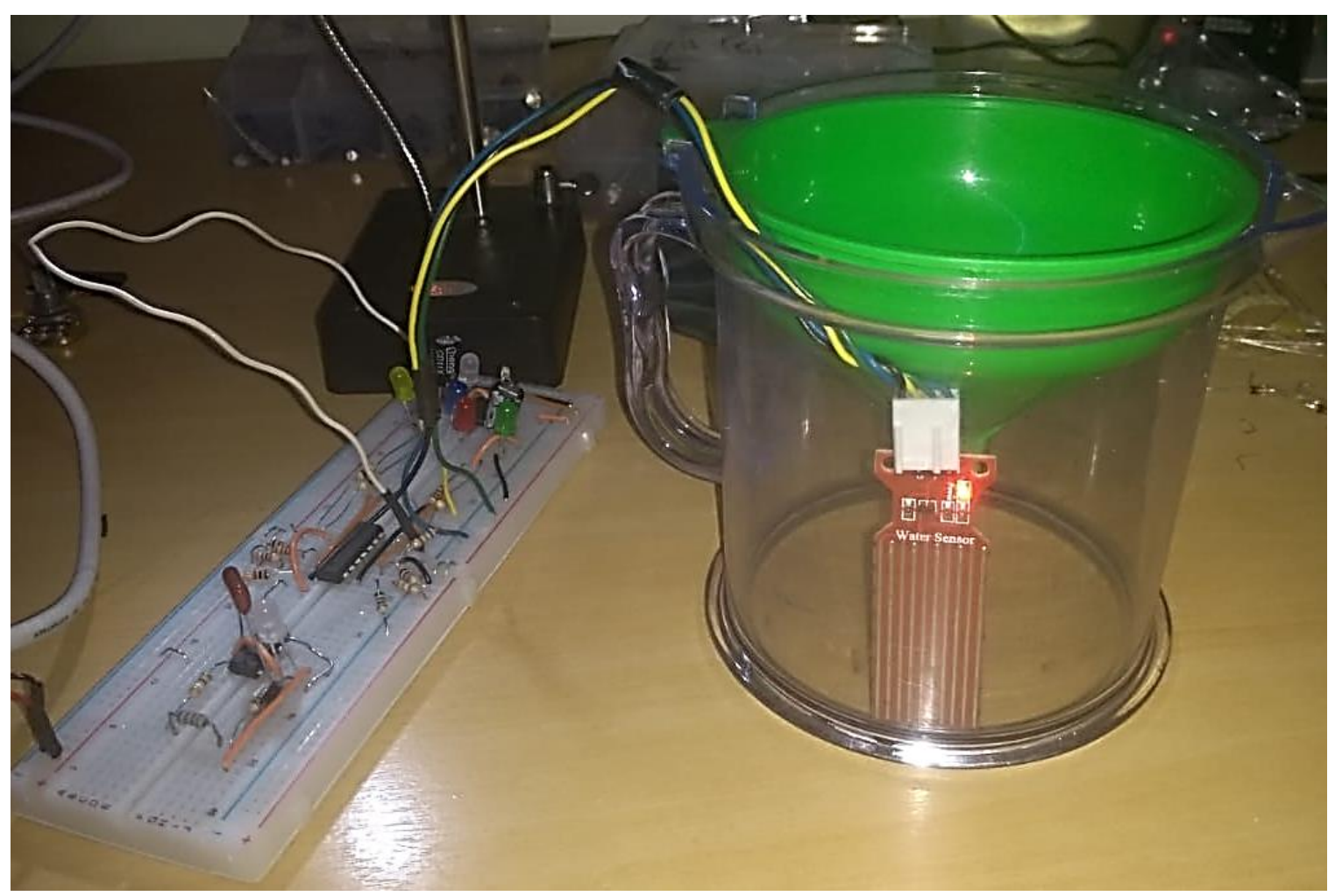

Fonte: Autores

Apesar da deficiência apresentada pelo sensor de nível, o circuito eletrônico do pluviômetro apresentou excelentes resultados. Conforme mostrado na Figura 26, o sensor foi substituído por um potenciômetro $22 \mathrm{k} \Omega$ para efeito de simulação e teste do nível pluviométrico no recipiente. É 
possível estabelecer níveis de tensão mais precisos com auxílio de resistores variáveis nas entradas de referência dos comparadores de tensão.

\section{CONSIDERAÇÕES FINAIS}

O presente estudo mostrou que protótipo desenvolvido e implementado apresentou resultado satisfatório, porém alguns problemas foram detectados, como por exemplo, a leitura do sensor de nível FD10 com pouca discrepância nas medidas, para os níveis a partir de $20 \mathrm{~mm}$, o que pode acarretar acionamentos desnecessários no sistema, sendo necessário para segurança da informação, a utilização de um transdutor de pressão em paralelo ao sensor de nível, para obtenção de medidas mais precisas. Também é possível afirmar que o comparador de tensão LM339 atendeu bem às expectativas quanto ao seu funcionamento, porém foi necessário realizar a adaptação dos ajustes do sinal de referência do circuito. O circuito impresso e o sistema mecânico de evacuação do recipiente se mostraram com formatação viável para utilização em campo, devido ao seu custo e tamanho compacto.

Como sugestões de trabalhos futuros, assim como possibilidades de otimização do dispositivo, cita-se:

- Implementação de sensores com maior abrangência e precisão, por exemplo: O sensor de nível de líquidos e Tape da Milone Technologies;

- Flexibilização de ajustes dos níveis de criticidade, através de chaves seletoras ou potenciômetros;

- Implementação de um sistema de contagem e monitoramento digital à distância;

- $\quad$ A utilização de um módulo Shield SIM908, para utilização em áreas remotas;

- Sistema de alimentação e carregamento de baterias por fontes de energia limpa;

- Calcular o custo do consumo e tendências de mercado.

Dessa forma, acredita-se que o protótipo desenvolvido teve seus objetivos alcançados, além de poder contribuir para o desenvolvimento de trabalhos futuros nesse segmento.

\section{REFERÊNCIAS}

ALEXANDRE, R. G.; UEHARA, D. M.; REDA, A. L. L. CALIBRAÇÃO DOS ERROS DE MEDIÇÃO DE PLUVIÔMETRO AUTOMÁTICO DE BÁSCULAS PARA CHUVAS INTENSAS NO BRASIL. Centro Universitário do Instituto Mauá de Tecnologia, Escola de Engenharia, São Caetano do Sul, 2006. 
ARAUJO ELIAS, A. A.; GONÇALVES, R. N. ARDWEATHER: UMA ESTAÇÃO

METEOROLÓGICA BASEADA NO ARDUINO E EM WEB SERVICES RESTFUL. $\boldsymbol{X I V}$ Safety, Health and Environment World Congress, Cubatão, 23 julho 2014.

ASSIS , F. D. [S.1.]: [s.n.].

BATISTEL, ; CAUVILLA, V. B.; LODI, M. R. DESEMPENHO DE UM PLUVIÔMETRO DIGITAL EM CAMPO. Associação Brasileira de Recursos Hídricos, Florianópolis, novembro 2017. 7.

ENGELBRECHT, A. ; Dolif G. ; JATOBA, A. ; Dias A. ; CARVALHO, P. V. R. ; GOMES, J. O. ; NOBRE A. C. . A Análise do Trabalho dos Meteorologistas na Previsão de Chuvas Intensas no Rio de Janeiro.. In: XVI Congresso Brasileiro de Ergonomia - ABERGO 2010, 2010, Rio de Janeiro. Anais do XVI Congresso Brasileiro de Ergonomia - ABERGO 2010. Rio de Janeiro: Associação brasileira de Ergonomia - ABERGO, 2010.

FERREIRA, W. J.; FISCH, G. COMPARATIVO ENTRE DADOS DE PRECIPITAÇÃO OBTIDOS POR PLUVIÔMETROS TIPPING BUCKET RAIN GAUGES E PLUVIÔMMETRO VILLE DE PARIS EM TAUBATÉ/SP. XI Mostra de Pós-Graduação-UNITAU, Taubaté, 2010.

MARCUZZO, F. F. N.; CARDOSO, R. D. Estudo Temporal e Espacialização Mensal e Anual das Chuvas na Parte Brasileira. Universidade Federal de Goiás. Goiânia, p. 10. 2012.

OSCAR JÚNIOR, A. C. D. S. Suscetibilidade ao impacto pluviométrico na região metropolitana do Rio de Janeiro: estudo de caso no município de Duque de Caxias. GEOUSP: Espaço e Tempo, online, v. 22, n. 1, p. 210-226, mês 2018. 